\title{
Absence of eigenvalues of two-dimensional magnetic Schrödinger operators
}

\author{
Luca Fanelli, David Krejčiř́k and Luis Vega
}

a) Dipartimento di Matematica, SAPIENZA Università di Roma, P. le Aldo Moro 5, 00185 Roma; fanelli@mat.uniroma1.it.

b) Department of Mathematics, Faculty of Nuclear Sciences and Physical Engineering, Czech Technical University in Prague, Trojanova 13, 12000 Prague 2, Czechia; david.krejcirik@fjfi.cvut.cz.

c) Departamento de Matemáticas, Universidad del Pais Vasco, Aptdo. 644, 48080 Bilbao, 8$\}$ Basque Center for Applied Mathematics (BCAM), Alameda Mazarredo 14, 48009 Bilbao, Spain; luis.vega@ehu.es8r lvega@bcamath.org.

17 October 2017

\begin{abstract}
By developing the method of multipliers, we establish sufficient conditions on the electric potential and magnetic field which guarantee that the corresponding two-dimensional Schrödinger operator possesses no point spectrum. The settings of complex-valued electric potentials and singular magnetic potentials of Aharonov-Bohm field are also covered.
\end{abstract}

\section{Introduction}

Given a vector potential $A: \mathbb{R}^{2} \rightarrow \mathbb{R}^{2}$ and a scalar potential $V: \mathbb{R}^{2} \rightarrow \mathbb{R}$, we consider the Schrödinger operator

$$
H_{A, V}:=(-i \nabla+A)^{2}+V \quad \text { in } \quad L^{2}\left(\mathbb{R}^{2}\right) .
$$

It is the quantum Hamiltonian of a non-relativistic electron interacting with the (vector) electric field $E=-\nabla V$ and the (scalar) magnetic field

$$
B=\partial_{1} A_{2}-\partial_{2} A_{1} .
$$

Writing $V=V_{+}-V_{-}$with $V_{ \pm}$being non-negative, we always assume that the negative part $V_{-}$of the electric potential $V$ is small in a suitable sense (see (4)), in order to make $H_{A, V}$ self-adjoint and bounded from below. More specifically, we understand $H_{A, V}$ as the Friedrichs extension of the symmetric operator (1) initially defined on smooth compactly supported functions.

The two-dimensional situation is special in the sense that $\mathbb{R}^{2}$ is the lowest dimensional Euclidean space for which the addition of the magnetic potential is non-trivial, while the unperturbed operator $H_{0,0}$ is still critical, i.e. unstable under small perturbations. In fact, it is well known that the purely electric operator $H_{0, V}$ possesses negative discrete eigenvalues whenever $V$ is negative and compactly supported, and there also exist examples of potentials generating positive eigenvalues embedded in the essential spectrum. At the same time, the spectrum of the purely magnetic operator $H_{A, 0}$ can be quite general, ranging from the purely essential spectrum $[0, \infty)$ for compactly supported magnetic field $B$ (Aharonov-Bohm solenoid), through the discrete set of infinitely degenerated eigenvalues (Landau levels) for constant $B \neq 0$, to purely discrete spectrum if $|B|$ diverges at infinity (magnetic bottles).

The objective of this paper is to identify physically relevant conditions which guarantee a total absence of eigenvalues of $H_{A, V}$. Because of the gauge invariance, these conditions should be given in terms of the physical quantity $B$ and not $A$. To state the desired result, we use the abbreviations $\nabla_{A}:=\nabla+i A$ for the magnetic gradient, $r(x):=|x|$ for the distance function from the origin and $\partial_{r} f(x):=\frac{x}{|x|} \cdot \nabla f(x)$ for the radial derivative of a function $f$. 
Theorem 1. Let $A \in L_{\mathrm{loc}}^{2}\left(\mathbb{R}^{2} ; \mathbb{R}^{2}\right)$ be such that $B \in L_{\mathrm{loc}}^{2}\left(\mathbb{R}^{2}\right)$. Suppose that $V \in L^{1}\left(\mathbb{R}^{2} ; \mathbb{R}\right)$ admits the decomposition $V=V^{(1)}+V^{(2)}$ with $V^{(1)} \in W_{\text {loc }}^{1,1}\left(\mathbb{R}^{2}\right)$ and $V^{(2)} \in L_{\text {loc }}^{2}\left(\mathbb{R}^{2}\right)$. Assume that there exist numbers $b, b_{1}, b_{2}, b_{3}, b_{4} \in[0,1)$ satisfying

$$
b_{1}+b_{2}^{2}+b_{3}^{2}+b_{4}<1
$$

such that, for all $\psi \in C_{0}^{\infty}\left(\mathbb{R}^{2}\right)$,

$$
\int_{\mathbb{R}^{2}} V_{-}(x)|\psi|^{2} \leq b^{2} \int_{\mathbb{R}^{2}}\left|\nabla_{A} \psi\right|^{2}
$$

and

$$
\begin{aligned}
\int_{\mathbb{R}^{2}} 4 r^{2}|B|^{2}|\psi|^{2} & \leq b_{1}^{2} \int_{\mathbb{R}^{2}}\left|\nabla_{A} \psi\right|^{2}, & & \int_{\mathbb{R}^{2}}\left[\partial_{r}\left(r V^{(1)}\right)\right]_{+}|\psi|^{2} \leq b_{2}^{2} \int_{\mathbb{R}^{2}}\left|\nabla_{A} \psi\right|^{2}, \\
\int_{\mathbb{R}^{2}}\left|V^{(2)}\right||\psi|^{2} & \leq b_{3}^{2} \int_{\mathbb{R}^{2}}\left|\nabla_{A} \psi\right|^{2}, & & \int_{\mathbb{R}^{2}} 4 r^{2}\left|V^{(2)}\right|^{2}|\psi|^{2} \leq b_{4}^{2} \int_{\mathbb{R}^{2}}\left|\nabla_{A} \psi\right|^{2},
\end{aligned}
$$

Then $H_{A, V}$ has no eigenvalues, i.e. $\sigma_{\mathrm{p}}\left(H_{A, V}\right)=\varnothing$.

The subordination condition (4) immediately implies that no non-positive number (including zero) can be an eigenvalue of $H_{A, V}$. The interest of the theorem lies in (5) with (3), which is a sufficient condition to avoid the existence of positive eigenvalues. The latter is clearly satisfied if $B=0, V^{(2)}=0$ and $\partial_{r}\left(r V^{(1)}\right) \leq 0$, where the last condition is a classical repulsiveness requirement. If $B \neq 0, V^{(2)} \neq 0$ or $\left[\partial_{r}\left(r V^{(1)}\right)\right]_{+} \neq 0$, however, it is not a priori clear that (5) is not void. In fact, if there is no magnetic field (i.e. $B \neq 0)$ and $V^{(2)} \neq 0$ or $\left[\partial_{r}\left(r V^{(1)}\right)\right]_{+} \neq 0$, the criticality of the two-dimensional free Hamiltonian $H_{0,0}$ implies that (5) cannot be satisfied (a similar statement holds for (4) with $V_{-} \neq 0$ ). If $V^{(2)} \neq 0$ or $\left[\partial_{r}\left(r V^{(1)}\right)\right]_{+} \neq 0$, it is therefore necessary that there is a magnetic field to exclude the existence of eigenvalues via Theorem 1 . The following proposition particularly ensures that (5) is generally not void.

Proposition 1. If there exist numbers $b, b_{1}, b_{2}, b_{3}, b_{4} \in[0,1)$ such that

$$
V_{-} \leq \pm b^{2} B
$$

and

$$
\begin{aligned}
4 r^{2}|B|^{2} & \leq \pm b_{1}^{2} B, & {\left[\partial_{r}\left(r V^{(1)}\right)\right]_{+} } & \leq \pm b_{2}^{2} B \\
\left|V^{(2)}\right| & \leq \pm b_{3}^{2} B, & 4 r^{2}\left|V^{(2)}\right|^{2} & \leq \pm b_{4}^{2} B
\end{aligned}
$$

with either a plus or minus sign, then (41) and (5) hold.

Proof. The claim follows from the well-known lower bound

$$
\forall \psi \in C_{0}^{\infty}\left(\mathbb{R}^{2}\right), \quad \int_{\mathbb{R}^{2}}\left|\nabla_{A} \psi\right|^{2} \geq \int_{\mathbb{R}^{2}} \pm B|\psi|^{2}
$$

which holds with either of the signs \pm (see, e.g., [1]).

For instance, if $V=0$ and the magnetic field $B$ is of definite sign, sufficiently small in the supremum norm and with a sufficiently small support, then (6) and (7), and therefore (4) and (5), hold.

Remark 1. Another source of sufficient conditions to guarantee (4) and (5) are in principal magnetic Hardy-type inequalities, reflecting the subcriticality of $H_{A, 0}$ whenever $B \neq 0$. For continuous $A$ satisfying the transverse gauge $x \cdot A(x)=0$ and locally integrable $B$, the Laptev-Weidl magnetic Hardy-type inequality (see [7]) states

$$
\forall \psi \in C_{0}^{\infty}\left(\mathbb{R}^{2}\right), \quad \int_{\mathbb{R}^{2}}\left|\nabla_{A} \psi(x)\right|^{2} \mathrm{~d} x \geq \int_{\mathbb{R}^{2}} \frac{\operatorname{dist}\left(\Phi_{B}(x), \mathbb{Z}\right)^{2}}{|x|^{2}}|\psi(x)|^{2} \mathrm{~d} x,
$$

where

$$
\Phi_{B}(x):=\frac{1}{2 \pi} \int_{\{|\xi| \leq|x|\}} B(\xi) \mathrm{d} \xi
$$


denotes the magnetic flux through the sphere of radius $|x|$ centred at the origin. Unfortunately, the pointwise condition $4|x|^{2}|B(x)|^{2} \leq|x|^{-2} \operatorname{dist}\left(\Phi_{B}(x), \mathbb{Z}\right)^{2}$, to guarantee the first inequality of (5) via (8), can never be satisfied for all sufficiently small $|x|$. From (8) one can deduce the bound

$$
\forall \psi \in C_{0}^{\infty}\left(\mathbb{R}^{2}\right), \quad \int_{\mathbb{R}^{2}}\left|\nabla_{A} \psi(x)\right|^{2} \mathrm{~d} x \geq \tilde{c}_{B} \int_{\mathbb{R}^{2}} \frac{|\psi(x)|^{2}}{1+|x|^{2}} \mathrm{~d} x
$$

where $\tilde{c}_{B}$ is a constant, which is positive if and only if the total magnetic flux $\lim _{|x| \rightarrow \infty} \Phi_{B}(x)$ is not an integer. See also [8] for other types of magnetic Hardy-type inequalities. As the most recent result, the following global Hardy-type inequality was derived in [3] for any smooth $A$

$$
\forall \psi \in C_{0}^{\infty}\left(\mathbb{R}^{2}\right), \quad \int_{\mathbb{R}^{2}}\left|\nabla_{A} \psi(x)\right|^{2} \mathrm{~d} x \geq c_{B} \int_{\mathbb{R}^{2}} \frac{|\psi(x)|^{2}}{1+|x|^{2} \log ^{2}|x|} \mathrm{d} x,
$$

where $c_{B}$ is a constant, which is positive if and only if $B$ is not identically equal to zero. The constant $c_{B}$ is given in terms of the first Neumann eigenvalue of the magnetic Laplacian in a disk and it is not clear whether a bound of the type (10) can actually provide a useful sufficient condition to guarantee (5). In any case, such a condition clearly forces $B$ to decay sufficiently fast at infinity.

Our strategy to establish Theorem 1 is based on the method of multipliers developed in the spectral context for Schrödinger operators in three and higher dimensions in our preceding paper [4. The restriction to the higher dimensions in [4] was caused by the essential usage of the classical Hardy inequality in the proof. The present paper is enabled by the observation that while the classical Hardy inequality is valid in three and higher dimensions only, it can be effectively replaced by the aforementioned magnetic Hardy-type inequalities in two dimensions and still yields a result in the context of multipliers. (We are grateful to Timo Weidl for the initial impetus to think about this extension.) Since the proof of Theorem 1 requires some important modifications of the original ideas of 4 ] and condition (5) differs from the form of sufficient conditions established in [4, we have decided to present this two-dimensional result to the community.

The organisation of this paper is as follows. Theorem 1 is proved in Section 2 , where we simultaneously present its higher-dimensional analogue (Theorem 2). In Section 3 we discuss the possibility of extending the present results to complex-valued electric potentials; in addition to the case of general regular fields (Theorems 3 and 4), we establish results for the singular Aharonov-Bohm potential (Theorem 5 ).

\section{The proof in any dimension}

We proceed in any dimension $d \geq 1$. At the same time, following 4 , we also allow the electric potential $V$ to be complex-valued. Physically, the imaginary part of $V$ can be interpreted as an energy gain/loss in an open quantum system.

Let us therefore assume $A \in L_{\mathrm{loc}}^{2}\left(\mathbb{R}^{d} ; \mathbb{R}^{d}\right)$ and $V \in L_{\mathrm{loc}}^{1}\left(\mathbb{R}^{d} ; \mathbb{C}\right)$. In any dimension, the physically relevant quantity is the $d$-covariant magnetic tensor

$$
B^{*}:=\nabla A-(\nabla A)^{T}
$$

and we assume $B^{*} \in L_{\text {loc }}^{2}\left(\mathbb{R}^{d} ; \mathbb{R}^{d \times d}\right)$. If $d=1$ this quantity is always equal to zero, so it is reasonable to exclude the one-dimensional situation from further considerations, but formally it is covered in the following. The magnetic field $B$ is the $(d-2)$-contravariant tensor obtained from $B^{*}$ as its Hodge dual. We therefore arrive at the scalar field (2) for $d=2$ and at the usual vector field $B=\operatorname{rot} A$ for $d=3$. We refer to 3 for more details on the formalism of the magnetic field in any dimension.

Let us consider the quadratic form

$$
h_{A, V}[\psi]:=\int\left|\nabla_{A} \psi\right|^{2}+\int V|\psi|^{2}, \quad \mathrm{D}\left(h_{A, V}\right):={\overline{C_{0}^{\infty}\left(\mathbb{R}^{d}\right)}}^{\|\cdot\| \mid}
$$

where

$$
\left\|\left.\psi\left|\|^{2}:=\int\right| \nabla_{A} \psi\right|^{2}+\int \Re V_{+}|\psi|^{2}+\int|\psi|^{2}\right.
$$


Here and in the sequel we abbreviate $\int:=\int_{\mathbb{R}^{d}}$ and omit the arguments of integrated functions. Under the assumption that there exist numbers $a_{1}, a_{2} \in[0,1)$ such that, for every $\psi \in C_{0}^{\infty}\left(\mathbb{R}^{d}\right)$,

$$
\int \Re V_{-}|\psi|^{2} \leq a_{1}^{2} \int\left|\nabla_{A} \psi\right|^{2} \quad \text { and } \quad \int|\Im V||\psi|^{2} \leq a_{2}^{2} \int\left|\nabla_{A} \psi\right|^{2}
$$

the form $h_{A, V}$ is sectorial and closed. Let us denote by $H_{A, V}$ the m-sectorial operator associated with $h_{A, V}$ via the first representation theorem (cf. [6. Thm. VI.2.1]). We have $\mathrm{D}\left(H_{A, V}\right) \subset \mathrm{D}\left(h_{A, V}\right) \subset$ $W^{1,2}\left(\mathbb{R}^{d}\right)$. Here the last inclusion employs the diamagnetic inequality

$$
\forall \psi \in W_{\mathrm{loc}}^{1,2}\left(\mathbb{R}^{d}\right), \quad\left|\nabla_{A} \psi\right| \geq|\nabla| \psi||,
$$

which we shall frequently use in the sequel. (If $\Im V=0$, then (13) coincides with (4) and in this case the operator $H_{A, V}$ is self-adjoint and bounded from below.)

In view of (13), a vertex and a semi-angle of $h_{A, V}$ are given by 0 and $\pi / 4$, respectively. Consequently,

$$
\sigma\left(H_{A, V}\right) \subset\{\lambda \in \mathbb{C}: \Re \lambda \geq|\Im \lambda|\}
$$

and in particular $H_{A, V}$ has no complex eigenvalue $\lambda$ with $\Re \lambda<|\Im \lambda|$. Actually, also the presence of zero eigenvalue can be excluded by (13) with help of (14).

Hence, it remains to exclude the existence of eigenvalues of $H_{A, V}$ in the sector on the right-hand side of (15). To this purpose, we employ the following crucial lemma.

Lemma 1. Let $A \in L_{\text {loc }}^{2}\left(\mathbb{R}^{d} ; \mathbb{R}^{d}\right)$ be such that $B^{*} \in L_{\text {loc }}^{2}\left(\mathbb{R}^{d}, \mathbb{R}^{d \times d}\right)$. Suppose that $V \in L^{1}\left(\mathbb{R}^{d} ; \mathbb{C}\right)$ admits the decomposition $\Re V=\Re V^{(1)}+\Re V^{(2)}$ with $\Re V^{(1)} \in W_{\text {loc }}^{1,1}\left(\mathbb{R}^{d}\right)$ and $\Re V^{(2)} \in L_{\text {loc }}^{2}\left(\mathbb{R}^{d}\right)$. Assume also (13). Let $u$ be a solution of $H_{A, V} u=\lambda u$ with $\Re \lambda \geq|\Im \lambda|$ satisfying

$$
\left(r^{2}\left|B_{\tau}^{*}\right|^{2}+\left[\partial_{r}\left(r \Re V^{(1)}\right)\right]_{+}+\left(1+r^{2}\right)\left|\Re V^{(2)}\right|^{2}+r^{2} \Im V+r \Re V_{-}+r^{-1}\right)|u|^{2} \in L^{1}\left(\mathbb{R}^{d}\right),
$$

where $B_{\tau}^{*}:=\frac{x}{|x|} \cdot B^{*}$. Then also $\left(r\left|\nabla_{A} u^{-}\right|+\left[\partial_{r}\left(r \Re V^{(1)}\right)\right]_{-}+r \Re V_{+}\right) \in L^{1}\left(\mathbb{R}^{d}\right)$ and the identity

$$
\begin{aligned}
\int\left|\nabla_{A} u^{-}\right|^{2}+\frac{|\Im \lambda|}{(\Re \lambda)^{1 / 2}}\left(\int r\left|\nabla_{A} u^{-}\right|^{2}-\frac{d-1}{2} \int \frac{\left|u^{-}\right|^{2}}{r}+\int r \Re V\left|u^{-}\right|^{2}\right) \\
=-2 \Im \int r B_{\tau}^{*} \cdot u^{-} \overline{\nabla_{A} u^{-}}+\int \partial_{r}\left(r \Re V^{(1)}\right)\left|u^{-}\right|^{2}+(1-d) \int \Re V^{(2)}\left|u^{-}\right|^{2} \\
\quad-2 \Re \int r \Re V^{(2)} u^{-\overline{\partial_{r}^{A} u^{-}}}+2 \Im \int r \Im V u^{-} \overline{\partial_{r}^{A} u^{-}}
\end{aligned}
$$

holds true (if $\lambda=0$ then the term multiplied by $|\Im \lambda| /(\Re \lambda)^{1 / 2}$ is not present), where $\partial_{r}^{A} f(x):=\frac{x}{|x|} \cdot \nabla_{A} f(x)$ and

$$
u^{ \pm}(x):=e^{ \pm i \operatorname{sgn}(\Im \lambda)(\Re \lambda)^{1 / 2}|x|} u(x) .
$$

Proof. The identity can be derived by following the method of multipliers developed in 4 based on previous ideas of [5] and [2]. Since the lemma is not explicitly stated in [4, we sketch the proof.

The eigenvalue equation $H_{A, V} u=\lambda u$ means that $u \in \mathrm{D}\left(H_{A, V}\right)$ and

$$
\forall v \in \mathrm{D}\left(h_{A, V}\right), \quad-\int \overline{\nabla_{A} v} \nabla_{A} u+\lambda \int \bar{v} u=\int \bar{v} V u
$$

Let $G_{1}, G_{2}, G_{3}: \mathbb{R}^{d} \rightarrow \mathbb{R}$ be three smooth functions. Choosing $v:=G_{1} u$ in (18), taking the real part of the obtained identity and integrating by parts, we obtain

$$
\Re \lambda \int G_{1}|u|^{2}-\int G_{1}\left|\nabla_{A} u\right|^{2}+\frac{1}{2} \int \Delta G_{1}|u|^{2}=\int G_{1} \Re V|u|^{2} .
$$

Analogously, choosing $v:=G_{2} u$ in (18), taking the imaginary part of the obtained identity and integrating by parts, we obtain

$$
\Im \lambda \int G_{2}|u|^{2}-\Im \int \nabla G_{2} \cdot \bar{u} \nabla_{A} u=\int G_{2} \Im V|u|^{2}
$$


Finally, choosing $v:=\left[\Delta_{A}, G_{3}\right]=2 \nabla G_{3} \cdot \nabla_{A} u+\Delta G_{3} u$ in (18) where $\Delta_{A}:=\nabla_{A} \cdot \nabla_{A}$ is the magnetic Laplacian, taking the real part of the obtained identity, integrating by parts and multiplying the result by $-1 / 2$, we obtain

$$
\begin{aligned}
\int \nabla_{A} u \cdot \nabla^{2} G_{3} \cdot \overline{\nabla_{A} u}-\frac{1}{4} \int \Delta^{2} G_{3}|u|^{2}+\Im \lambda \Im \int & \nabla G_{3} \cdot u \overline{\nabla_{A} u}+\Im \int \nabla G_{3} \cdot B^{*} \cdot u \overline{\nabla_{A} u} \\
& =-\frac{1}{2} \int \Delta G_{3} \Re V|u|^{2}-\Re \int \nabla G_{3} \cdot V u \overline{\nabla_{A} u}
\end{aligned}
$$

where $\nabla^{2} G_{3}$ denotes the Hessian matrix of $G_{3}$ and $\Delta^{2}:=\Delta \Delta$ is the bi-Laplacian. Identity (17) is obtained by combining (19)-(21) with special choices of the multipliers:

$$
\begin{array}{r}
{\left[(19) \text { with } G_{1}(x):=1\right]+\left[(20) \text { with } G_{2}(x):=2 \Re \lambda \operatorname{sgn}(\Im \lambda)|x|\right]+\left[(21) \text { with } G_{3}(x):=|x|^{2}\right]} \\
-\left[(19) \text { with } G_{1}(x):=\frac{|\Im \lambda|}{(\Re \lambda)^{1 / 2}}|x|\right] .
\end{array}
$$

(If $\lambda=0$ then the last subtraction is not performed.) Here the main idea is to replace $u$ by $u^{-}$using the identities

$$
\left|\nabla_{A} u^{-}(x)\right|=\left|\nabla_{A} u(x)-i(\Re \lambda)^{1 / 2} \operatorname{sgn}(\Im \lambda) \frac{x}{|x|} u(x)\right| \quad \text { and } \quad B_{\tau}^{*} \cdot \bar{u} \nabla_{A} u=B_{\tau}^{*} \cdot \overline{u^{-}} \nabla_{A} u^{-},
$$

where the latter employs the fact that $B_{\tau}^{*}$ is tangential, i.e. $x \cdot B_{\tau}^{*}(x)=0$.

Up to now, the procedure explained above has been purely formal, because we a priori do not know that the individual integrals converge. To make it rigorous, one can follow [4 and replace $u$ by approximating solutions by using a standard cutoff and mollification argument. In this way, one arrives at an approximating version of (17) and the desired identity is obtained after passing to the limit in the cutoff and mollification parameters, by employing the convergence of a set of integrals expressed by (16).

Now let us come back to the initial hypothesis that $V$ is real-valued. Then $H_{A, V}$ is self-adjoint, necessarily $\Im \lambda=0$ and it remains to exclude the existence of non-negative eigenvalues. In this case, (17) reduces to

$$
\begin{aligned}
\int\left|\nabla_{A} u^{-}\right|^{2}= & -2 \Im \int r B_{\tau}^{*} \cdot u^{-} \overline{\nabla_{A} u^{-}}+\int \partial_{r}\left(r V^{(1)}\right)\left|u^{-}\right|^{2} \\
& +(1-d) \int V^{(2)}\left|u^{-}\right|^{2}-2 \Re \int r V^{(2)} u^{-} \overline{\partial_{r}^{A} u^{-}} .
\end{aligned}
$$

Using the Schwarz inequality, we have

$$
\begin{aligned}
\int\left|\nabla_{A} u^{-}\right|^{2} \leq & 2 \sqrt{\int r^{2}\left|B_{\tau}^{*}\right|^{2}\left|u^{-}\right|^{2}} \sqrt{\int\left|\nabla_{A} u^{-}\right|^{2}}+\int\left[\partial_{r}\left(r V^{(1)}\right)\right]_{+}\left|u^{-}\right|^{2} \\
& +|1-d| \int\left|V^{(2)}\right|\left|u^{-}\right|^{2}+2 \sqrt{\int r^{2}\left|V^{(2)}\right|^{2}\left|u^{-}\right|^{2}} \sqrt{\int\left|\nabla_{A} u^{-}\right|^{2}} \\
\leq & \left(b_{1}+b_{2}^{2}+(d-1) b_{3}^{2}+b_{4}\right) \int\left|\nabla_{A} u^{-}\right|^{2},
\end{aligned}
$$

where the last inequality follows by conditions

$$
\begin{aligned}
\int 4 r^{2}\left|B_{\tau}^{*}\right|^{2}|\psi|^{2} & \leq b_{1}^{2} \int\left|\nabla_{A} \psi\right|^{2}, & \int\left[\partial_{r}\left(r V^{(1)}\right)\right]_{+}|\psi|^{2} & \leq b_{2}^{2} \int\left|\nabla_{A} \psi\right|^{2}, \\
\int\left|V^{(2)}\right||\psi|^{2} & \leq b_{3}^{2} \int\left|\nabla_{A} \psi\right|^{2}, & \int 4 r^{2}\left|V^{(2)}\right|^{2}|\psi|^{2} & \leq b_{4}^{2} \int\left|\nabla_{A} \psi\right|^{2},
\end{aligned}
$$

assumed to be valid for every $\psi \in C_{0}^{\infty}\left(\mathbb{R}^{d}\right)$. Making the hypothesis

$$
b_{1}+b_{2}^{2}+(d-1) b_{3}^{2}+b_{4}<1
$$


we conclude with $\nabla_{A} u^{-}=0$. By the diamagnetic inequality (14), it follows that $u^{-}=0$ and thus $u=0$. Consequently, the eigenvalue equation $H_{A, V} u=\lambda u$ for $\lambda \geq 0$ admits only trivial solutions. It concludes the proof that the point spectrum of $H_{A, V}$ is empty.

Let us summarise the multidimensional result into the following theorem.

Theorem 2. Let $A \in L_{\text {loc }}^{2}\left(\mathbb{R}^{d} ; \mathbb{R}^{d}\right)$ be such that $B^{*} \in L_{\text {loc }}^{2}\left(\mathbb{R}^{d} ; \mathbb{R}^{d \times d}\right)$. Suppose that $V \in L^{1}\left(\mathbb{R}^{d} ; \mathbb{R}\right)$ admits the decomposition $V=V^{(1)}+V^{(2)}$ where $V^{(1)} \in W_{\mathrm{loc}}^{1,1}\left(\mathbb{R}^{d}\right)$ and $V^{(2)} \in L_{\mathrm{loc}}^{2}\left(\mathbb{R}^{d}\right)$. Assume that there exist numbers $a, b_{1}, b_{2}, b_{3}, b_{4} \in[0,1)$ satisfying (25) such that (13) and (24) hold. Then $H_{A, V}$ has no eigenvalues, i.e. $\sigma_{\mathrm{p}}\left(H_{A, V}\right)=\varnothing$.

Theorem 1 is a special case for $d=2$. Notice that $\left|B_{\tau}^{*}\right|=|B|$ if $d=2$.

\section{Extensions to complex-valued electric potentials}

After establishing the crucial identity of Lemma 1, one of the next steps of [4 to deal with it was to use a weighted Hardy inequality

$$
\forall \psi \in C_{0}^{\infty}\left(\mathbb{R}^{d}\right), \quad \int r|\nabla \psi|^{2} \geq \frac{(d-1)^{2}}{4} \int \frac{|\psi|^{2}}{r}
$$

together with the diamagnetic inequality (14) and to replace the first two terms in the round brackets on the left-hand side of (17) by the lower bound

$$
\int r\left|\nabla_{A} u^{-}\right|^{2}-\frac{d-1}{2} \int \frac{\left|u^{-}\right|^{2}}{r} \geq \frac{d-3}{d-1} \int r\left|\nabla_{A} u^{-}\right|^{2} .
$$

Although (26) is valid also for $d=2$, the lower bound (27) is negative, which spoils the subsequent argument ( $c f$. the procedure above Theorem 2). This is the reason why it is not immediate, in two dimensions, to use formula (17) for ensuring the absence of eigenvalues with non-zero imaginary part (unless $H_{A, V}$ is self-adjoint). Notice, however, that a condition excluding real eigenvalues even if $H_{A, V}$ is non-self-adjoint is easy to obtain in the same way as above, because then the troublesome term represented by the round brackets on the left-hand side of (17) is not present.

Here we present several alternative ways how to use (17) in order to guarantee the total absence of eigenvalues even if $H_{A, V}$ is not self-adjoint. Since the alternative approaches are not needed in higher dimensions, in this section we again restrict to the two-dimensional situation.

First of all, we rewrite (17) as follows $(d=2)$ :

$$
\begin{aligned}
\int\left|\nabla_{A} u^{-}\right|^{2}+\frac{|\Im \lambda|}{(\Re \lambda)^{1 / 2}}\left(\int r\left|\nabla_{A} u^{-}\right|^{2}-\frac{1}{2} \int \frac{\left|u^{-}\right|^{2}}{r}+\int r \Re V_{+}\left|u^{-}\right|^{2}\right) \\
=-2 \Im \int r B_{\tau}^{*} \cdot u^{-} \overline{\nabla_{A} u^{-}}+\int \partial_{r}\left(r \Re V^{(1)}\right)\left|u^{-}\right|^{2}-\int \Re V^{(2)}\left|u^{-}\right|^{2} \\
\quad-2 \Re \int r \Re V^{(2)} u^{-} \overline{\partial_{r}^{A} u^{-}}+2 \Im \int r \Im V u^{-} \overline{\partial_{r}^{A} u^{-}}+\frac{|\Im \lambda|}{(\Re \lambda)^{1 / 2}} \int r \Re V_{-}\left|u^{-}\right|^{2},
\end{aligned}
$$

i.e. we keep on the left-hand side just the positive part of $\Re V$. As above, the terms multiplied by $|\Im \lambda| /(\Re \lambda)^{1 / 2}$ are not present if $\lambda=0$. More generally, if $\lambda=0$ or $\Im \lambda=0$, the identity (28) coincides with (23) and the sufficient condition of Theorem 1 already implies the absence of such eigenvalues. Below we derive worth sufficient conditions to cover the case of complex eigenvalues as well.

\subsection{Employing the positivity of the real part of the electric field}

An obvious condition to make the round brackets on the left-hand side of (28) non-negative is to require

$$
\frac{1}{2} \int \frac{|\psi|^{2}}{r} \leq \int r\left|\nabla_{A} \psi\right|^{2}+\int r \Re V_{+}|\psi|^{2}
$$

for every $\psi \in C_{0}^{\infty}\left(\mathbb{R}^{d}\right)$. Recalling (26), it can be satisfied provided that we require for instance the pointwise bound

$$
\Re V_{+} \geq \frac{1}{4 r^{2}} .
$$


Having ensured the non-negativity of the round brackets on the left-hand side of (28), the terms on the right-hand side can be handled as above. It is only important to comment on how to get rid of the energy dependent fraction at the last term on the right-hand side of (28). We proceed as follows:

$$
\begin{aligned}
\frac{|\Im \lambda|}{(\Re \lambda)^{1 / 2}} \int r \Re V_{-}\left|u^{-}\right|^{2} & \leq \frac{|\Im \lambda|}{(\Re \lambda)^{1 / 2}} \sqrt{\int r^{2}\left|\Re V_{-}\right|^{2}\left|u^{-}\right|^{2}} \sqrt{\int\left|u^{-}\right|^{2}} \\
& \leq \frac{|\Im \lambda|^{1 / 2}}{(\Re \lambda)^{1 / 2}} \sqrt{\int r^{2}\left|\Re V_{-}\right|^{2}\left|u^{-}\right|^{2}} \sqrt{\int|\Im V|\left|u^{-}\right|^{2}} \\
& \leq \sqrt{\int r^{2}\left|\Re V_{-}\right|^{2}\left|u^{-}\right|^{2}} \sqrt{\int|\Im V|\left|u^{-}\right|^{2}}
\end{aligned}
$$

where the first estimate is due the Schwarz inequality, the second estimate follows from (20) with a constant choice for the multiplier $G_{2}$ and the last estimate is implied by the restriction to the sector (15). Consequently, from (28) we deduce the bound

$$
\begin{aligned}
\int\left|\nabla_{A} u^{-}\right|^{2} \leq & 2 \sqrt{\int r^{2}|B|^{2}\left|u^{-}\right|^{2}} \sqrt{\int\left|\nabla_{A} u^{-}\right|^{2}}+\int\left[\partial_{r}\left(r \Re V^{(1)}\right)\right]_{+}\left|u^{-}\right|^{2} \\
& +\int\left|\Re V^{(2)}\right|\left|u^{-}\right|^{2}+2 \sqrt{\int r^{2}\left|\Re V^{(2)}\right|^{2}\left|u^{-}\right|^{2}} \sqrt{\int\left|\nabla_{A} u^{-}\right|^{2}} \\
& +2 \sqrt{\int r^{2}|\Im V|^{2}\left|u^{-}\right|^{2}} \sqrt{\int\left|\nabla_{A} u^{-}\right|^{2}}+\sqrt{\int r^{2}\left|\Re V_{-}\right|^{2}\left|u^{-}\right|^{2}} \sqrt{\int|\Im V|\left|u^{-}\right|^{2}} . \\
\leq & \left(b_{1}+b_{2}^{2}+b_{3}^{2}+b_{4}+b_{5}+b_{6} a_{2}\right) \int\left|\nabla_{A} u^{-}\right|^{2}
\end{aligned}
$$

where the last inequality follows by (13) and conditions

$$
\begin{array}{rlrl}
\int 4 r^{2}|B|^{2}|\psi|^{2} & \leq b_{1}^{2} \int\left|\nabla_{A} \psi\right|^{2}, & & \int\left[\partial_{r}\left(r \Re V^{(1)}\right)\right]_{+}|\psi|^{2} \leq b_{2}^{2} \int\left|\nabla_{A} \psi\right|^{2}, \\
\int\left|\Re V^{(2)}\right||\psi|^{2} & \leq b_{3}^{2} \int\left|\nabla_{A} \psi\right|^{2}, & \int 4 r^{2}\left|\Re V^{(2)}\right|^{2}|\psi|^{2} \leq b_{4}^{2} \int\left|\nabla_{A} \psi\right|^{2}, \\
\int 4 r^{2}|\Im V||\psi|^{2} \leq b_{5}^{2} \int\left|\nabla_{A} \psi\right|^{2}, & \int r^{2}\left|\Re V_{-}\right|^{2}|\psi|^{2} \leq b_{6}^{2} \int\left|\nabla_{A} \psi\right|^{2},
\end{array}
$$

assumed to be valid for every $\psi \in C_{0}^{\infty}\left(\mathbb{R}^{d}\right)$. Making the hypothesis

$$
b_{1}+b_{2}^{2}+b_{3}^{2}+b_{4}+b_{5}+b_{6} a_{2}<1 \text {, }
$$

we conclude with $u=0$ as above.

We summarise the result of this subsection in the following theorem.

Theorem 3. Let $A \in L_{\mathrm{loc}}^{2}\left(\mathbb{R}^{2} ; \mathbb{R}^{2}\right)$ be such that $B \in L_{\mathrm{loc}}^{2}\left(\mathbb{R}^{2} ; \mathbb{R}\right)$. Suppose that $V \in L^{1}\left(\mathbb{R}^{2} ; \mathbb{C}\right)$ admits the decomposition $\Re V=\Re V^{(1)}+\Re V^{(2)}$ where $\Re V^{(1)} \in W_{\mathrm{loc}}^{1,1}\left(\mathbb{R}^{d}\right)$ and $\Re V^{(2)} \in L_{\mathrm{loc}}^{2}\left(\mathbb{R}^{d}\right)$. Assume that there exist numbers $a_{1}, a_{2}, b_{1}, b_{2}, b_{3}, b_{4}, b_{5}, b_{6} \in[0,1)$ satisfying (33) such that (13) and (32) hold. Moreover, assume (29). Then $H_{A, V}$ has no eigenvalues, i.e. $\sigma_{\mathrm{p}}\left(H_{A, V}\right)=\varnothing$.

Remark 2. Alternatively, keeping the last term from the right-hand side of (28) in the round brackets on left-hand side, one can require the condition

$$
\frac{1}{2} \int \frac{|\psi|^{2}}{r}+\int r \Re V_{-}|\psi|^{2} \leq \int r\left|\nabla_{A} \psi\right|^{2}+\int r \Re V_{+}|\psi|^{2}
$$

instead of (29). Recalling (26), it can be satisfied provided that we require for instance the pointwise bound

$$
\Re V \geq \frac{1}{4 r^{2}} .
$$

Then the conditions on the last line of (32) can be ignored and one can take $b_{5}, b_{6}=0$ in (33). 


\subsection{A more robust approach}

Denote by $D_{R}:=\left\{x \in \mathbb{R}^{2}:|x|<R\right\}$ the open disk of radius $R>0$. The main ingredient of this subsection is the following Hardy-Poincaré-type inequality.

Lemma 2. One has

$$
\forall \psi \in W_{0}^{1,2}\left(D_{R}\right), \quad \int_{D_{R}}|\nabla \psi(x)|^{2} \mathrm{~d} x \geq \frac{1}{4 R} \int_{D_{R}} \frac{|\psi(x)|^{2}}{|x|} \mathrm{d} x .
$$

Proof. For every $f \in C^{1}([0, R])$ such that $f(R)=0$, we have

$$
\begin{aligned}
\int_{0}^{R} \frac{|f(r)|^{2}}{r} r \mathrm{~d} r & =\int_{0}^{R}|f(r)|^{2} r^{\prime} \mathrm{d} r \\
& =-2 \int_{0}^{R} \Re\left[\overline{f(r)} f^{\prime}(r)\right] r \mathrm{~d} r \\
& \leq 2 \sqrt{\int_{0}^{R}\left|f^{\prime}(r)\right|^{2} r \mathrm{~d} r} \sqrt{\int_{0}^{R}|f(r)|^{2} r \mathrm{~d} r} \\
& \leq 2 R \sqrt{\int_{0}^{R}\left|f^{\prime}(r)\right|^{2} r \mathrm{~d} r} \sqrt{\int_{0}^{R} \frac{|f(r)|^{2}}{r}} r \mathrm{~d} r
\end{aligned}
$$

and therefore

$$
\int_{0}^{R}\left|f^{\prime}(r)\right|^{2} r \mathrm{~d} r \geq \frac{1}{4 R} \int_{0}^{R} \frac{|f(r)|^{2}}{r} r \mathrm{~d} r .
$$

This one-dimensional inequality implies (34) after expressing the gradient in spherical coordinates and by neglecting the angular component.

Given two positive numbers $R_{1}<R_{2}$, let $\eta:[0, \infty) \rightarrow[0,1]$ be such that $\eta=1$ on $\left[0, R_{1}\right], \eta=0$ on $\left[R_{2}, \infty\right)$ and $\eta(r)=\left(R_{2}-r\right) /\left(R_{2}-R_{1}\right)$ for $r \in\left(R_{1}, R_{2}\right)$. We denote by the same symbol $\eta$ the radial function $\eta \circ r: \mathbb{R}^{2} \rightarrow[0,1]$. Now, writing $u^{-}=\eta u^{-}+(1-\eta) u^{-}$and using Lemma 2, we estimate the troublesome term of (28) as follows:

$$
\begin{aligned}
\int \frac{\left|u^{-}\right|^{2}}{r} & \leq 2 \int \frac{\left|\nabla u^{-}\right|^{2}}{r}+2 \int \frac{\left|(1-\eta) u^{-}\right|^{2}}{r} \\
& \leq 8 R_{2} \int\left|\nabla\left(\eta u^{-}\right)\right|^{2}+\frac{2}{R_{1}} \int\left|u^{-}\right|^{2} \\
& \leq 16 R_{2} \int\left|\nabla u^{-}\right|^{2}+\frac{16 R_{2}}{\left(R_{2}-R_{1}\right)^{2}} \int\left|u^{-}\right|^{2}+\frac{2}{R_{1}} \int\left|u^{-}\right|^{2} .
\end{aligned}
$$

Choosing $R_{2}:=\epsilon(\Re \lambda)^{1 / 2} /|\Im \lambda|$ and $R_{1}:=R_{2} / 2$ with any positive number $\epsilon$, we get

$$
\begin{aligned}
\frac{|\Im \lambda|}{(\Re \lambda)^{1 / 2}} \int \frac{\left|u^{-}\right|^{2}}{r} & \leq 16 \epsilon \int\left|\nabla u^{-}\right|^{2}+\frac{68}{\epsilon} \frac{|\Im \lambda|^{2}}{\Re \lambda} \int\left|u^{-}\right|^{2} \\
& \leq 16 \epsilon \int\left|\nabla u^{-}\right|^{2}+\frac{68}{\epsilon} \frac{|\Im \lambda|}{\Re \lambda} \int|\Im V|\left|u^{-}\right|^{2} \\
& \leq 16 \epsilon \int\left|\nabla u^{-}\right|^{2}+\frac{68}{\epsilon} \int|\Im V|\left|u^{-}\right|^{2},
\end{aligned}
$$

where the second estimate follows from (20) with a constant choice for the multiplier $G_{2}$ and the last estimate is implied by the restriction to the sector (15). Using additionally (26), we thus deduce from (28) the crucial inequality

$$
\begin{aligned}
(1-4 \epsilon) & \int\left|\nabla_{A} u^{-}\right|^{2}-\frac{17}{\epsilon} \int|\Im V|\left|u^{-}\right|^{2}+\frac{|\Im \lambda|}{(\Re \lambda)^{1 / 2}} \int r \Re V_{+}\left|u^{-}\right|^{2} \\
\leq & -2 \Im \int r B_{\tau}^{*} \cdot u^{-} \overline{\nabla_{A} u^{-}}+\int \partial_{r}\left(r \Re V^{(1)}\right)\left|u^{-}\right|^{2}-\int \Re V^{(2)}\left|u^{-}\right|^{2} \\
& -2 \Re \int r \Re V^{(2)} u^{-} \overline{\partial_{r}^{A} u^{-}}+2 \Im \int r \Im V u^{-} \overline{\partial_{r}^{A} u^{-}}+\frac{|\Im \lambda|}{(\Re \lambda)^{1 / 2}} \int r \Re V_{-}\left|u^{-}\right|^{2} .
\end{aligned}
$$


Now, putting the second term from the left-hand side of (35) to the right-hand side, neglecting the last term on the left-hand side and treating the terms on the right-hand side as in Section 3.1 (see particularly (31)), we get

$$
\begin{aligned}
(1-4 \epsilon) \int\left|\nabla_{A} u^{-}\right|^{2} \leq & 2 \sqrt{\int r^{2}|B|^{2}\left|u^{-}\right|^{2}} \sqrt{\int\left|\nabla_{A} u^{-}\right|^{2}}+\int\left[\partial_{r}\left(r \Re V^{(1)}\right)\right]_{+}\left|u^{-}\right|^{2} \\
& +\int\left|\Re V^{(2)}\right|\left|u^{-}\right|^{2}+2 \sqrt{\int r^{2}\left|\Re V^{(2)}\right|^{2}\left|u^{-}\right|^{2}} \sqrt{\int\left|\nabla_{A} u^{-}\right|^{2}} \\
& +2 \sqrt{\int r^{2}|\Im V|^{2}\left|u^{-}\right|^{2}} \sqrt{\int\left|\nabla_{A} u^{-}\right|^{2}}+\sqrt{\int r^{2}\left|\Re V_{-}\right|^{2}\left|u^{-}\right|^{2}} \sqrt{\int|\Im V|\left|u^{-}\right|^{2}} \\
& +\frac{17}{\epsilon} \int|\Im V|\left|u^{-}\right|^{2} \\
\leq & \left(b_{1}+b_{2}^{2}+b_{3}^{2}+b_{4}+b_{5}+b_{6} a_{2}+\frac{17}{\epsilon} a_{2}^{2}\right) \int\left|\nabla_{A} u^{-}\right|^{2},
\end{aligned}
$$

where the last inequality follows by (13) and conditions (32). Making the hypothesis

$$
b_{1}+b_{2}^{2}+b_{3}^{2}+b_{4}+b_{5}+b_{6} a_{2}+\frac{17}{\epsilon} a_{2}^{2}+4 \epsilon<1
$$

we therefore conclude with $u=0$ as above.

We summarise the result of this subsection in the following theorem.

Theorem 4. Let $A \in L_{\text {loc }}^{2}\left(\mathbb{R}^{2} ; \mathbb{R}^{2}\right)$ be such that $B \in L_{\text {loc }}^{2}\left(\mathbb{R}^{2} ; \mathbb{R}\right)$. Suppose that $V \in L^{1}\left(\mathbb{R}^{2} ; \mathbb{C}\right)$ admits the decomposition $\Re V=\Re V^{(1)}+\Re V^{(2)}$ where $\Re V^{(1)} \in W_{\mathrm{loc}}^{1,1}\left(\mathbb{R}^{d}\right)$ and $\Re V^{(2)} \in L_{\mathrm{loc}}^{2}\left(\mathbb{R}^{d}\right)$. Assume that there exist numbers $\epsilon, a_{1}, a_{2}, b_{1}, b_{2}, b_{3}, b_{4}, b_{5}, b_{6} \in[0,1)$ satisfying (33) such that (13) and (32) hold. Then $H_{A, V}$ has no eigenvalues, i.e. $\sigma_{\mathrm{p}}\left(H_{A, V}\right)=\varnothing$.

\subsection{The Aharonov-Bohm potential}

Finally, we consider the special case of the singular Aharonov-Bohm potential

$$
A(x):=(-\sin \theta, \cos \theta) \frac{\alpha(\theta)}{r},
$$

where $\left(x_{1}, x_{2}\right)=(r \cos \theta, r \sin \theta)$ is the parameterisation via polar coordinates, $r \in(0, \infty), \theta \in[0,2 \pi)$, and $\alpha:[0,2 \pi) \rightarrow \mathbb{R}$ is an arbitrary bounded function. In this case, the magnetic field $B$ equals zero everywhere except for $x=0$. In fact

$$
B=2 \pi \bar{\alpha} \delta
$$

in the sense of distributions, where $\delta$ is the Dirac delta function and

$$
\bar{\alpha}:=\frac{1}{2 \pi} \int_{0}^{2 \pi} \alpha(\theta) \mathrm{d} \theta
$$

has the physical meaning of the total magnetic flux. We notice that $A$ can be gauged out whenever $\bar{\alpha}$ is an integer. To measure the strength of the Aharonov-Bohm field, we introduce the distance of the total magnetic flux $\bar{\alpha}$ to the set of integers

$$
\beta:=\operatorname{dist}(\bar{\alpha}, \mathbb{Z})
$$

and assume $\bar{\alpha} \notin \mathbb{Z}$, so that $\beta \in(0,1 / 2]$.

Since $A \notin L_{\text {loc }}^{2}\left(\mathbb{R}^{2} ; \mathbb{R}^{2}\right)$, the Aharonov-Bohm potential does not satisfy our standing regularity assumption. In the case (38), we still understand $H_{A, V}$ as the operator associated with the form (11), but now with the form core $C_{0}^{\infty}\left(\mathbb{R}^{2}\right)$ being replaced by $C_{0}^{\infty}\left(\mathbb{R}^{2} \backslash\{0\}\right)$. To get an m-sectorial operator, we assume the validity of (13) for all $\psi \in C_{0}^{\infty}\left(\mathbb{R}^{2} \backslash\{0\}\right)$ now. If $\beta=0$, then $H_{A, V}$ is unitarily equivalent to the magnetic-free operator $H_{0, V}$. 
Following the proof of Lemma 1, it can be shown that the identity

$$
\begin{aligned}
& \int\left|\nabla_{A} u^{-}\right|^{2}+\frac{|\Im \lambda|}{(\Re \lambda)^{1 / 2}}\left(\int r\left|\nabla_{A} u^{-}\right|^{2}-\frac{1}{2} \int \frac{\left|u^{-}\right|^{2}}{r}+\int r \Re V_{+}\left|u^{-}\right|^{2}\right) \\
& =\int \partial_{r}\left(r \Re V^{(1)}\right)\left|u^{-}\right|^{2}-\int \Re V^{(2)}\left|u^{-}\right|^{2} \\
& \quad-2 \Re \int r \Re V^{(2)} u^{-} \overline{\partial_{r}^{A} u^{-}}+2 \Im \int r \Im V u^{-} \overline{\partial_{r}^{A} u^{-}}+\frac{|\Im \lambda|}{(\Re \lambda)^{1 / 2}} \int r \Re V_{-}\left|u^{-}\right|^{2}
\end{aligned}
$$

holds, provided that the solution of $H_{A, V} u=\lambda u$ satisfies conditions (16) without the first term (containing $B_{\tau}^{*}$ ). Formally, (41) follows after plugging (39) into (17).

The principal idea of this subsection is that the singular magnetic Hardy-type inequality due to Laptev and Weidl (see [7, Thm. 3])

$$
\forall \psi \in C_{0}^{\infty}\left(\mathbb{R}^{2} \backslash\{0\}\right), \quad \int_{\mathbb{R}^{2}}\left|\nabla_{A} \psi(x)\right|^{2} \mathrm{~d} x \geq \beta^{2} \int_{\mathbb{R}^{2}} \frac{|\psi(x)|^{2}}{|x|^{2}} \mathrm{~d} x
$$

holds true, where $\beta$ is introduced in (40). We also use its weighted variant included in the following lemma, which is a magnetic improvement upon (26).

Lemma 3. Let $A$ be given by (38). Then one has

$$
\forall \psi \in C_{0}^{\infty}\left(\mathbb{R}^{2} \backslash\{0\}\right), \quad \int_{\mathbb{R}^{2}}|x|\left|\nabla_{A} \psi(x)\right|^{2} \mathrm{~d} x \geq\left(\frac{1}{4}+\beta^{2}\right) \int_{\mathbb{R}^{2}} \frac{|\psi(x)|^{2}}{|x|} \mathrm{d} x .
$$

Proof. Passing to polar coordinates, we have

$$
\begin{aligned}
\int_{\mathbb{R}^{2}}|x|\left|\nabla_{A} \psi(x)\right|^{2} \mathrm{~d} x & =\int_{0}^{2 \pi} \int_{0}^{\infty}\left|\partial_{r} \phi(r, \theta)\right|^{2} r^{2} \mathrm{~d} r \mathrm{~d} \theta+\int_{0}^{2 \pi} \int_{0}^{\infty}\left|\left(\partial_{\theta}+i \alpha(\theta)\right) \phi(r, \theta)\right|^{2} r^{2} \mathrm{~d} r \mathrm{~d} \theta \\
& \geq \frac{1}{4} \int_{0}^{2 \pi} \int_{0}^{\infty}|\phi(r, \theta)|^{2} r^{2} \mathrm{~d} r \mathrm{~d} \theta+\beta^{2} \int_{0}^{2 \pi} \int_{0}^{\infty}|\phi(r, \theta)|^{2} r^{2} \mathrm{~d} r \mathrm{~d} \theta
\end{aligned}
$$

where $\phi(r, \theta):=\psi(r \cos \theta, r \sin \theta)$. Here the first integral on the right-hand side is estimated by a classical weighted one-dimensional Hardy inequality (which is actually behind the proof of (26) ), while the bound on the second integral employs that the first eigenvalue of the operator $\left[-i \partial_{\theta}+\alpha(\theta)\right]^{2}$ in $L^{2}((0,2 \pi))$, subject to periodic boundary conditions, equals $\beta^{2}$.

Lemma 3 enables us to handle the troublesome term on the left-hand side of (41) as follows. Given any positive number $\delta$, we write

$$
\begin{aligned}
-\int r\left|\nabla_{A} u^{-}\right|^{2}+\frac{1}{2} \int \frac{\left|u^{-}\right|^{2}}{r} & \leq\left(\frac{1}{4}-\beta^{2}\right) \int \frac{\left|u^{-}\right|^{2}}{r} \\
& =\left(\frac{1}{4}-\beta^{2}\right) \int_{D_{\delta}} \frac{\left|u^{-}\right|^{2}}{r}+\left(\frac{1}{4}-\beta^{2}\right) \int_{\mathbb{R}^{2} \backslash \overline{D_{\delta}}} \frac{\left|u^{-}\right|^{2}}{r} \\
& \leq\left(\frac{1}{4}-\beta^{2}\right) \delta \int \frac{\left|u^{-}\right|^{2}}{r^{2}}+\left(\frac{1}{4}-\beta^{2}\right) \frac{1}{\delta} \int\left|u^{-}\right|^{2} \\
& \leq\left(\frac{1}{4}-\beta^{2}\right) \delta \int \frac{\left|u^{-}\right|^{2}}{r^{2}}+\left(\frac{1}{4}-\beta^{2}\right) \frac{1}{\delta|\Im \lambda|} \int|\Im V|\left|u^{-}\right|^{2} \\
& \leq\left(\frac{1}{4}-\beta^{2}\right) \frac{\delta}{\beta^{2}} \int\left|\nabla_{A} u^{-}\right|^{2}+\left(\frac{1}{4}-\beta^{2}\right) \frac{1}{\delta|\Im \lambda|} \int|\Im V|\left|u^{-}\right|^{2},
\end{aligned}
$$

where the first inequality is due to (43), estimates in the second inequality are elementary, the third estimate follows from (20) with a constant choice for the multiplier $G_{2}$ and the last inequality employs (42). Choosing $\delta:=\beta^{2} \epsilon(\Re \lambda)^{1 / 2} /|\Im \lambda|$ with any positive $\epsilon$ and using (15), we therefore get

$$
\frac{|\Im \lambda|}{(\Re \lambda)^{1 / 2}}\left(-\int r\left|\nabla_{A} u^{-}\right|^{2}+\frac{1}{2} \int \frac{\left|u^{-}\right|^{2}}{r}\right) \leq\left(\frac{1}{4}-\beta^{2}\right) \epsilon \int\left|\nabla_{A} u^{-}\right|^{2}+\left(\frac{1}{4}-\beta^{2}\right) \frac{1}{\beta^{2} \epsilon} \int|\Im V|\left|u^{-}\right|^{2} .
$$


Neglecting the last term on the left-hand side of (41) and treating the other terms as in the first inequality of (36), we arrive at

$$
\begin{aligned}
\int\left|\nabla_{A} u^{-}\right|^{2} \leq & \int\left[\partial_{r}\left(r \Re V^{(1)}\right)\right]_{+}\left|u^{-}\right|^{2} \\
& +\int\left|\Re V^{(2)}\right|\left|u^{-}\right|^{2}+2 \sqrt{\int r^{2}\left|\Re V^{(2)}\right|^{2}\left|u^{-}\right|} \sqrt{\int\left|\nabla_{A} u^{-}\right|^{2}} \\
& +2 \sqrt{\int r^{2}|\Im V|^{2}\left|u^{-}\right|^{2}} \sqrt{\int\left|\nabla_{A} u^{-}\right|^{2}}+\sqrt{\int r^{2}\left|\Re V_{-}\right|^{2}\left|u^{-}\right|^{2}} \sqrt{\int|\Im V|\left|u^{-}\right|^{2}} \\
& +\left(\frac{1}{4}-\beta^{2}\right) \epsilon \int\left|\nabla_{A} u^{-}\right|^{2}+\left(\frac{1}{4}-\beta^{2}\right) \frac{1}{\beta^{2} \epsilon} \int|\Im V|\left|u^{-}\right|^{2} \\
\leq & {\left[b_{2}^{2}+b_{3}^{2}+b_{4}+b_{5}+b_{6} a_{2}+\left(\frac{1}{4}-\beta^{2}\right) \epsilon+\left(\frac{1}{4}-\beta^{2}\right) \frac{1}{\beta^{2} \epsilon} a_{2}^{2}\right] \int\left|\nabla_{A} u^{-}\right|^{2} . }
\end{aligned}
$$

Here the last inequality follows by (13) and conditions (32) that are assumed to be valid for all $\psi \in$ $C_{0}^{\infty}\left(\mathbb{R}^{2} \backslash\{0\}\right)$. The first integral in (32) is interpreted as zero, so that this condition is always satisfied for the Aharonov-Bohm field (38). Making the hypothesis

$$
b_{2}^{2}+b_{3}^{2}+b_{4}+b_{5}+b_{6} a_{2}+\left(\frac{1}{4}-\beta^{2}\right) \epsilon+\left(\frac{1}{4}-\beta^{2}\right) \frac{1}{\beta^{2} \epsilon} a_{2}^{2}<1,
$$

we therefore conclude with $u=0$ as above.

We summarise the result of this subsection in the following theorem.

Theorem 5. Let the vector potential $A$ be given by (38) with $\bar{\alpha} \notin \mathbb{Z}$ and suppose that the scalar potential $V \in L^{1}\left(\mathbb{R}^{2} ; \mathbb{C}\right)$ admits the decomposition $\Re V=\Re V^{(1)}+\Re V^{(2)}$ where $\Re V^{(1)} \in W_{\text {loc }}^{1,1}\left(\mathbb{R}^{d}\right)$ and $\Re V^{(2)} \in$ $L_{\mathrm{loc}}^{2}\left(\mathbb{R}^{d}\right)$. Assume that there exist numbers $\epsilon, a_{1}, a_{2}, b_{2}, b_{3}, b_{4}, b_{5}, b_{6} \in[0,1)$ satisfying (44) such that (13) and (32) hold for all $\psi \in C_{0}^{\infty}\left(\mathbb{R}^{2} \backslash\{0\}\right)$. Then $H_{A, V}$ has no eigenvalues, i.e. $\sigma_{\mathrm{p}}\left(H_{A, V}\right)=\varnothing$.

In particular, in the electric-free case $V=0$, we recover the well known result that the AharonovBohm Laplacian $H_{A, 0}$ possesses no eigenvalues. Notice also that the sufficient conditions of Theorem 5 substantially simplify in the regime when the Aharonov-Bohm field is the strongest, i.e. $\beta=1 / 2$.

\section{Acknowledgments}

We are grateful to Timo Weidl for valuable suggestions. The research of D.K. was partially supported by FCT (Portugal) through project PTDC/MAT-CAL/4334/2014. The research of L.V. was partially supported by ERCEA Advanced Grant 669689-HADE, MTM2014-53145-P, and IT641-13.

\section{References}

[1] A. Balinsky, A. Laptev, and A. Sobolev, Generalized Hardy inequality for the magnetic Dirichlet forms, J. Stat. Phys. 116 (2004), 507-521.

[2] J. A. Barceló, L. Vega, and M. Zubeldia, The forward problem for the electromagnetic Helmholtz equation with critical singularities, Adv. Math. 240 (2013), 636-671.

[3] C. Cazacu and D. Krejčiřík, The Hardy inequality and the heat equation with magnetic field in any dimension, Comm. Partial Differential Equations 41 (2016), 1056-1088.

[4] L. Fanelli, D. Krejčiřík, and L. Vega, Spectral stability of Schrödinger operators with subordinated complex potentials, J. Spectr. Theory (2017), arXiv:1506.01617 [math.SP].

[5] T. Ikebe and Y. Saito, Limiting absorption method and absolute continuity for the Schrödinger operator, J. Math. Kyoto Univ. 12 (1972), 513-542.

[6] T. Kato, Perturbation theory for linear operators, Springer-Verlag, Berlin, 1966.

[7] A. Laptev and T. Weidl, Hardy inequalities for magnetic Dirichlet forms, Oper. Theory Adv. Appl. 108 (1999), 299-305.

[8] T. Weidl, A remark on Hardy type inequalities for critical Schrödinger operators with magnetic fields, Oper. Theory Adv. Appl. 110 (1999), 345-352. 